\title{
The Unpredictable Errors of Micro Tactile Metrology - Factors Affecting Stylus tip Contamination
}

\author{
P. Kinnell, R. Habeb \\ Institute for Advanced Manufacturing, The University of Nottingham, Nottingham NG7 2RD, UK \\ E-mail: Peter.Kinnell@Nottigham.ac.uk
}

In 3D tactile micro-metrology the contamination of probing devices is a major problem that affects the accuracy and repeatability of measured dimensions. Despite a large body of research in the field of micro CMM and micro probe design there is limited research which has been done so far to explain and tackle this problem. In this work, experimental probing on a range of materials using a micro coordinate measuring machine was conducted to investigate the mechanism of stylus tip contamination. In addition the effects of surface finish on the build-up of stylus tip contamination were also studied. The results provide practitioners with guidelines which allow for the likely build-up of stylus tip contamination to be minimized based on sample material type and surface finish.

Keywords: Micro-coordinate measurement machine, $\mu \mathrm{CMM}$, tactile probing, contaminant build-up.

\section{INTRODUCTION}

A $\mathrm{S}$ A RESULT of the widespread trend towards increased miniaturization and complexity of many modern products, the need for high-accuracy 3D metrology of complex geometries continues to grow. To meet this need there are a range of metrology instruments available that operate in both a non-contact manner such as variable focus, or interferometric microscopes; as well as systems such as stylus based instruments which operate in a contact mode.

While both non-contact and contact systems provide useful measurement results the complexity of modern micro products and components means that as yet there is not a single measurement principle that will meet all measurement challenges. For example, despite the many benefits of a noncontact metrology system, the measurement of highly reflective surfaces, transparent materials, high aspect ratio holes or trenches, or steep slopes are problematic for optical non-contact systems. Examples of products which may contain these types of features include MEMS (microelectro-mechanical-system) sensors, micro fluidic devices, micro-optics, micro-injection moulding tools, or engine injector nozzles.

To address this need a number of micro-coordinatemeasurement-machine $(\mu \mathrm{CMM})$ instruments have been developed and are commercially available [1], [2]. These systems are similar to conventional coordinatemeasurement-machines, but have been optimised to provided high resolution and accuracy over a small measurement volume (typically a $100 \mathrm{~mm}$ cube). They also make use of specialized micro probing systems, which consist of a tungsten stylus bonded to a silicon sensor. Bonded to the free end of the stylus is a ruby stylus tip with, diameters on the order of $100 \mu \mathrm{m}$ currently available [1]. These probing systems impart extremely low probing forces of typically less than $0.5 \mathrm{mN}$ [3].

The probing system used in this work (manufactured by Carl Zeiss, for use on the Zeiss F25 $\mu$ CMM) consists of a MEMS chip with a cross-shaped base (approximately $4 \mathrm{~mm}$ in length), a tungsten stylus (50 $\mu \mathrm{m}$ diameter) which is bonded to the MEMS chip, and a spherical ruby tip bonded to the other end of the stylus. The diameter of the stylus tips available for this probing system varies between 120 to $700 \mu \mathrm{m}$. To achieve the $250 \mathrm{~nm}$ uncertainty made possible by the Zeiss F25, the ruby-tip must be completely free of any contaminates. A particle of a few microns could cause significant dimensioning errors that are orders of magnitude greater than the expected error. For example when a new clean micro probing systems is used it will first be calibrated to determine the position and geometry of the stylus tip. Once the calibration is complete, the micro probing system may then be used to make accurate measurements. If the clean stylus tip is subsequently contaminated with debris during the probing operation, then there will be a possibility for errors to arise. Once the debris has been attached to the probe tip, any repeat measurements that coincide with this contact point on the sphere will result in a bias error approximately equal to the size of the contamination less any plastic or elastic deformation [11]. Based on this scenario a particle of just five microns in size may create a bias error that is 20 times greater than the expected system total uncertainty of $250 \mathrm{~nm}$.

There are a number of sources of contamination that may impact the performance of micro probing systems during service. For example: dust or atmospheric contaminates such as pollen or other organic debris may contaminate stylus tips; loose debris such as particulates on the artefact surface from previous manufacturing processes may be picked up by the stylus tip during operation; the action of probing may result in micro surface wear releasing particulates to contaminate the stylus tip [4]-[6]. The debris will adhere to the tip's surface as a result of surface attraction forces which become a dominant force on the micro scale [7]-[11].

While other systematic sources of error may be well characterized allowing valid levels of measurement uncertainty to be specified, the unpredictable nature of stylus tip contamination does not allow for this type of 
analysis. In previous work stylus tip contamination was addressed from a cleaning perspective; methods for stylus tip cleaning were investigated and promising methods for debris removal were presented [11]. However, this work did not investigate or quantify the likely timeframe during normal operation that would results in significant stylus tip contamination. This paper will present results from an initial study that aims to quantify the build-up of stylus tip debris under typical measurement procedures. The build-up of contamination during repeated single point probing and linescans will be studied for a range of materials.
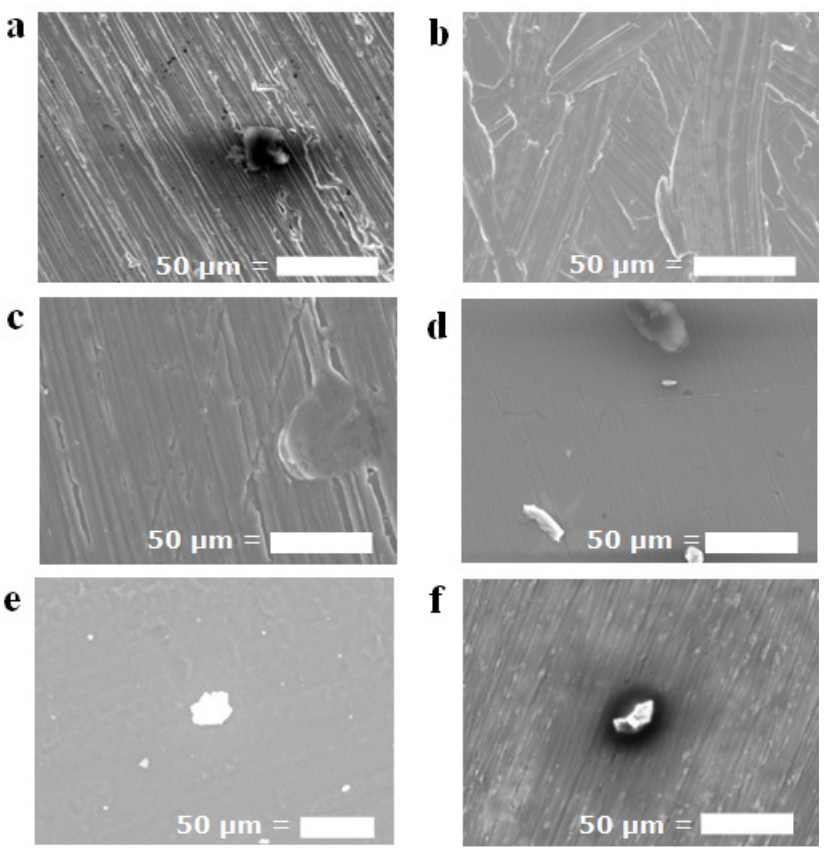

Fig.1. Micrographs showing examples of typical debris remaining on the sample surface prior to test probing, scale bars are $50 \mu \mathrm{m}$, a) Copper (smooth), b) Copper (rough) c) Aluminium d) Stainless Steel e) Nylon f) Titanium.

\section{Methodology}

To establish the factors affecting the build-up of stylus tip contamination a series of samples were first prepared these consisted of pieces of Aluminium, Copper, Nylon, Steel, and Titanium. The samples were selected as they represent a range of materials including both high strength materials such as Titanium, and Steel, as well as relatively low strength materials such as Copper, Nylon, and Aluminium. The surface roughness of each sample was measured using a Taylor-Hobson Intra surface profiler with a sample length of $5.1 \mathrm{~mm}$ and a Gaussian filter applied with a lower cut off of $0.008 \mathrm{~mm}$, and upper cut off of $0.8 \mathrm{~mm}$; these results are given in Table 1.

\subsection{Preparation of material samples}

In preparation for probing each material sample was properly cleaned using standard ultrasonic cleaning baths which are commonly used in the micro and precision manufacturing environment. Two cleaning solutions were used on each sample, first a water-based detergent was used to remove general contamination. Then a finial clean was carried out using clean iso-propanol. Samples were held for 15 minutes in each cleaning solution.

Following the cleaning cycle the samples were allowed to air-dry inside a clean container. An SEM (scanning electron microscope) was used to visualise the level of remaining debris on the surface of each sample after washing. While the samples were general well cleaned inspection with the SEM in back scattered electron imaging to highlight specimen density variations showed some evidence of debris of unknown origin still present on the samples. No further action was taken to remove these few surface particles, as this reflects the nature of a produced micro component following typical cleaning prior to metrology. SEM images of each of the samples clearly show debris present on some samples, as shown in Fig.1. In addition to these six samples three additional titanium samples with highly structured surfaces that were produced using a plain water jet erosion process resulting in significant surface roughness were also selected for analysis to investigate the extreme case of highly structured surfaces.

Table 1. Surface finish measurements for all samples.

\begin{tabular}{|l|l|l|l|l|}
\hline Sample & $\mathbf{R a} \boldsymbol{\mu m}$ & $\mathbf{R p} \boldsymbol{\mu m}$ & $\mathbf{R v} \boldsymbol{\mu m}$ & $\mathbf{R z} \boldsymbol{\mu m}$ \\
\hline Smooth copper & 0.17 & 0.85 & 0.58 & 1.42 \\
\hline Rough copper & 0.76 & 2.21 & 2.75 & 4.95 \\
\hline Plastic & 0.78 & 1.97 & 4.96 & 6.93 \\
\hline Aluminium & 0.25 & 0.92 & 1.06 & 1.98 \\
\hline Steel & 0.05 & 0.16 & 0.21 & 0.37 \\
\hline Titanium a & 0.09 & 0.36 & 0.66 & 1.02 \\
\hline Titanium b & 0.55 & 1.75 & 1.62 & 3.37 \\
\hline Titanium c & 10.52 & 28.07 & 30.74 & 58.81 \\
\hline Titanium d & 12.30 & 36.49 & 29.60 & 66.09 \\
\hline
\end{tabular}

\subsection{Preparation of the micro probe sensors}

The probe sensors used in this work were manufactured by Zeiss with $125 \mu \mathrm{m}$ ruby stylus tips, for use on the Zeiss F25 $\mu \mathrm{CMM}$. The probes are designed to impart a maximum contact for of $0.5 \mathrm{mN}$ during probing. Each probe sensor contains the full probing systems, including the probe sensor, stylus, and stylus tip. The probe sensors were each inspected using SEM to ensure the cleanness of the stylus tip, and note any pre-existing contamination or irregularities prior to probing.

\subsection{Probing tests plan}

After cleaning all samples and analysing the probe sensors, the probing tests were performed. Probing was executed in two modes, either single point measurements that were repeated at multiple locations on the sample surface, or repeated line scans of $2 \mathrm{~mm}$ over the sample surface. Care was taken to ensure probing was performed over the same local area of the samples, with probing constrained to an area of approximately $10 \times 10 \mathrm{~mm}$ in size. The number of probing operations was initially set to 20 points, then increased to 200 points, and then finally 1000 or 2000 points. The probing direction for the majority of samples was vertical such that the pole of the stylus tip 
surface was making contact with the sample and therefore debris was expected to adhere to this area of the stylus tip. However, as the availability of probe sensors was limited in some cases probing was performed on the sides of the stylus tip. Details are given within the results section with respect to the probing direction used for each sample. The probing directions are illustrated in Fig.2.

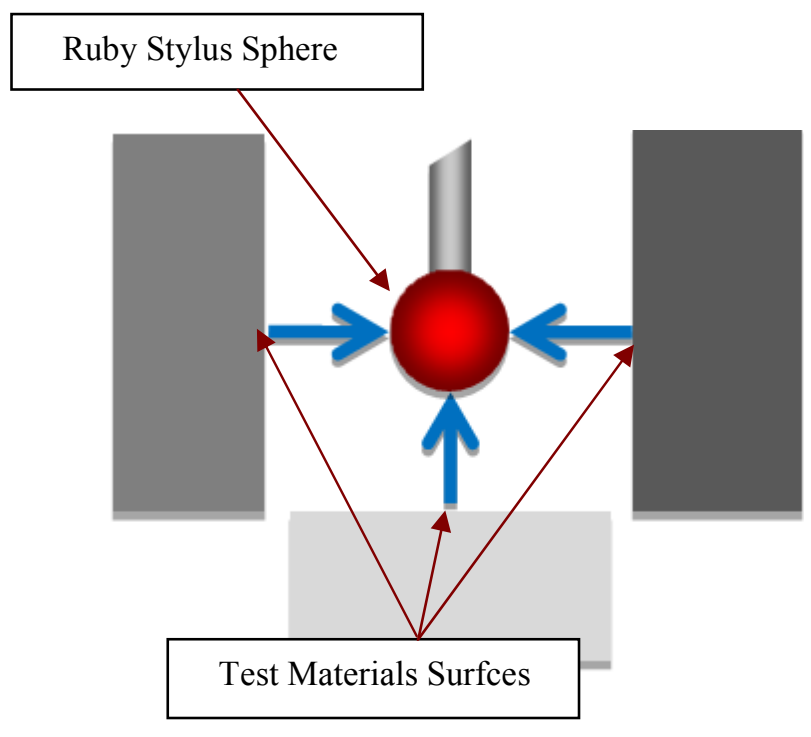

Fig.2. Probing directions used, with the arrows indication the typical contact points that test material makes on the probe surface, the majority of probing was conducted vertically such that the lower poll of the Ruby sphere was the contact point (indicated by the lowest arrow).

Between each set of probing tests, inspection of the stylus tip surface was performed to find out if any new debris was present on the tip surface. For quick detection of the nonconductive stylus tips which are made of ruby, BSE (back scattered emission) environmental SEM microscopy was used. This microscope was based in the same laboratory as the $\mu \mathrm{CMM}$ which minimized the potential for environmental contamination from airborne particulates. Following the initial inspection the stylus tips were transported in clean containers to a second laboratory for a material composition analysis of the observed debris on the tip surface. For this analysis used an environmental SEM with energy-dispersive X-ray spectroscopy (EDX) was implemented.

\section{STYLUS TIP CONTAMINATION STUDY RESULTS}

Probing of the prepared samples was performed in a number of configurations. The probing sequence followed for each sample, with a description of the surface contamination observed using SEM is given in Table 2. In addition a note is given to indicate the result of the EDX analysis.

For single point probing no debris was found until after the 1000 points of probing on both Copper samples. It was present following Nylon probing after 1000 points. The same probing system was then used to complete scan probing on the rough Copper sample, with $2 \mathrm{~mm}$ lines being scanned 20 times. Fig.3. shows environmental SEM images of the stylus tip in a clean state, and following contamination. A small protrusion can be seen on the equator of the probe. This was confirmed to be Aluminium Oxide using EDX. The origin of this defect is unknown, however, as it was present on a new unused micro probe sensor, and is the same material as the stylus tip it is assumed to be integral to the stylus tip. No other instances of Aluminium Oxide debris or similar protrusion were observed in the rest of the work. For both the pieces of debris shown in Fig.3b., EDX analysis was able to confirm the material compositions were consistent with Copper, and Nylon as indicated. A second new probe sensor was then used to scan the smooth copper sample. In this case debris was also visible on the stylus tip surface as shown in Fig. 4. EDX analysis of this debris was shown to be organic in nature with no indication of Copper, indicating that the stylus tip had picked up some unknown contamination following the scanning sequence on smooth Copper.

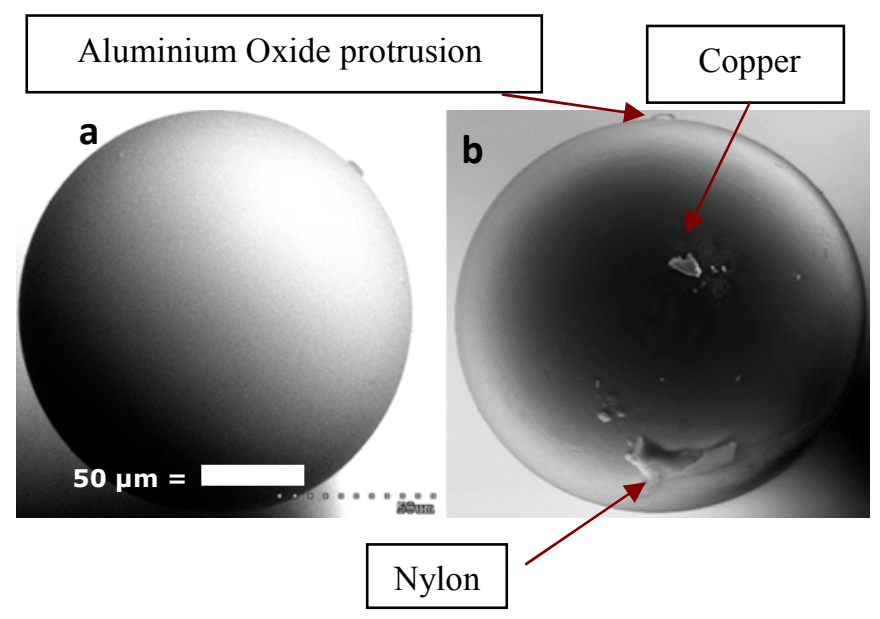

Fig.3. a) stylus tip in the new condition contamination free. A small protrusion can be seen on the horizon of stylus tip which is a defect integral to the ruby sphere. b) Indicates positions of stylus tip contamination.

The Copper (both smooth and rough) and Nylon samples were the first to be analysed. Initially single point probing was considered, starting from 20 probing points, and then the number of points scanned was increased to 200, and then finally 1000 points. Following each set of probing points the samples were observed using an environmental SEM.

A third new probe sensor was then used to probe the samples of aluminium, steel and titanium, as described in Table 1.

The SEM images of the stylus tip before and after probing can be seen in Fig.5., showing the three probing sites on the lower pole, as well as on the opposite sides of the stylus tip equator. EDX analysis of the visible debris was able to confirm the presence of aluminium, as well as traces of iron, but no Titanium was observed. Other elements found indicated organic debris that transferred from the samples onto the stylus tip. 
Table 2. Summary of probing experimental results, indicating probing sequence and observed stylus tip contamination debris following SEM inspection.

\begin{tabular}{|c|c|c|}
\hline Sample & Probing sequence & Stylus tip State \\
\hline $\begin{array}{l}\text { Smooth } \\
\text { copper }\end{array}$ & $\begin{array}{l}20 \text { points followed by } \\
\text { BSE detection. Repeat } \\
\text { at } 200 \& 1000 \text { points. } \\
\text { All at vertical direction } \\
\text { probing. Followed by } \\
20 \text { line scans, vertical } \\
\text { direction. }\end{array}$ & $\begin{array}{l}\text { None was found after } \\
\text { each set of probing } \\
\text { points. Debris found } \\
\text { following the scan } \\
\text { probing. EDX } \\
\text { confirmed not Copper }\end{array}$ \\
\hline $\begin{array}{l}\text { Rough } \\
\text { copper }\end{array}$ & As for Smooth Copper & $\begin{array}{l}\text { Same as for Smooth } \\
\text { Copper except EDX } \\
\text { confirmed Copper }\end{array}$ \\
\hline Nylon & $\begin{array}{l}20 \text { points followed by } \\
\text { BSE detection for } \\
\text { debris. Then repeat at } \\
200 \text { points and } 1000 \\
\text { points. All at vertical } \\
\text { probing direction }\end{array}$ & $\begin{array}{l}\text { Debris found after } \\
1000 \text { of probing } \\
\text { points, EDX } \\
\text { confirmed Nylon }\end{array}$ \\
\hline Aluminium & $\begin{array}{l}1000 \text { points (vertical } \\
\text { probing direction) }\end{array}$ & $\begin{array}{l}\text { Debris found, EDX } \\
\text { confirmed } \\
\text { Aluminium }\end{array}$ \\
\hline Steel & $\begin{array}{l}2000 \text { points (right side } \\
\text { probing direction) }\end{array}$ & $\begin{array}{l}\text { Debris found EDX } \\
\text { confirmed to contain } \\
\text { Iron }\end{array}$ \\
\hline Titanium a & $\begin{array}{l}1000 \text { points (left side } \\
\text { probing direction) }\end{array}$ & $\begin{array}{l}\text { Debris found EDX } \\
\text { confirmed not } \\
\text { Titanium. }\end{array}$ \\
\hline Titanium b & $\begin{array}{l}1000 \text { points, vertical } \\
\text { probing direction }\end{array}$ & $\begin{array}{l}\text { Debris found EDX } \\
\text { confirmed not } \\
\text { Titanium. }\end{array}$ \\
\hline Titanium c & $\begin{array}{l}1000 \text { points, vertical } \\
\text { probing direction }\end{array}$ & $\begin{array}{l}\text { Debris found EDX } \\
\text { confirmed not } \\
\text { Titanium. }\end{array}$ \\
\hline Titanium d & $\begin{array}{l}1000 \text { points, vertical } \\
\text { probing direction }\end{array}$ & $\begin{array}{l}\text { Debris found EDX } \\
\text { confirmed not } \\
\text { Titanium. }\end{array}$ \\
\hline
\end{tabular}

Fig.6. a) Stylus tip state following probing of Nylon and Copper samples. b) stylus tip state following probing of titanium samples $\mathrm{b}$, c, and d, with large quantities of additional contamination present.

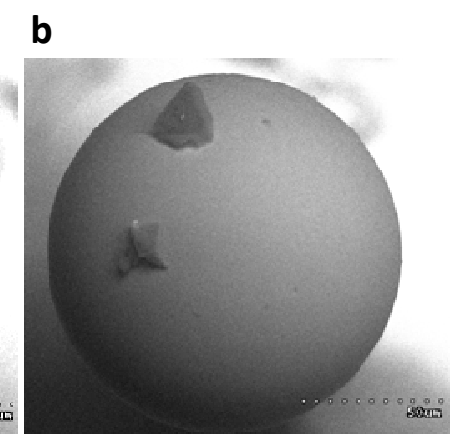

Fig.4. a) stylus tip in the new condition contamination free. b) indicates observed stylus tip contamination.

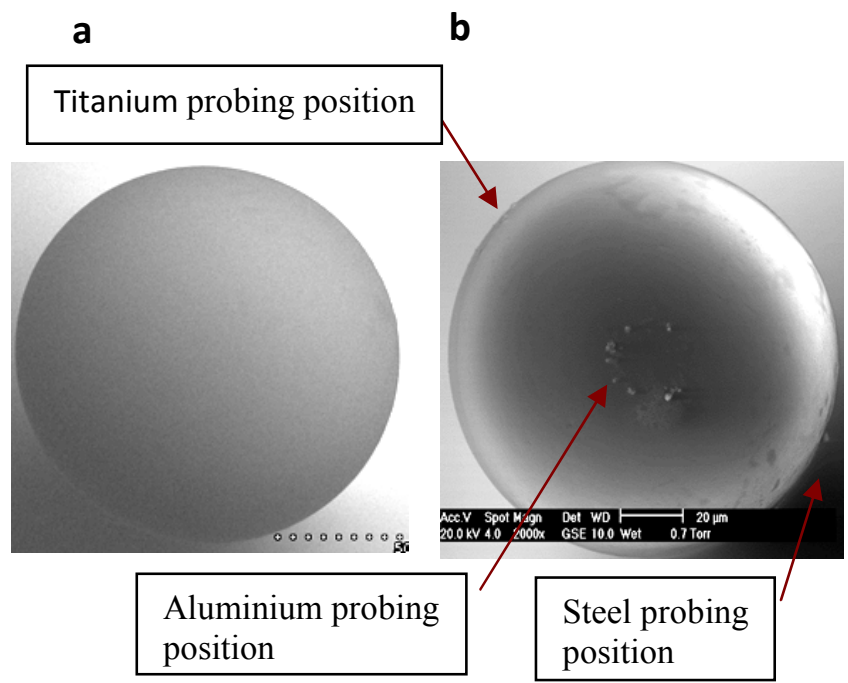

Fig.5. a) stylus tip in the new condition contamination free. b) Indicates observed stylus tip contamination positions following probing of Aluminium, Steel, and Titanium a samples.

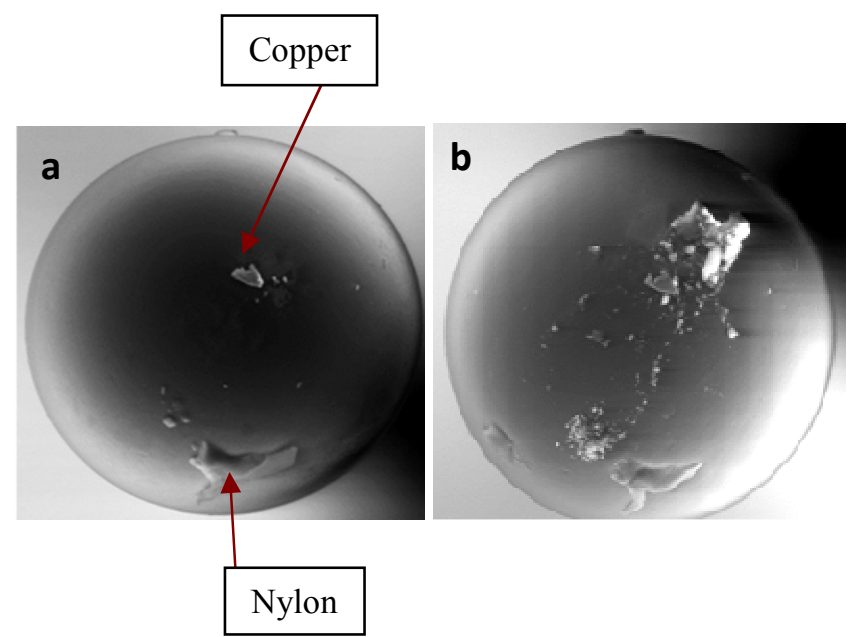


For the final probing experiment, the probe sensor first used to for Nylon and Copper probing as shown in Fig.3. was reused for probing of Titanium samples b, c, and d. In this case 1000 points was performed on each sample, with a steady build up of contamination resulting in the stylus tip state as shown in Fig.6. Fig.6a. shows the state of the stylus tip before the titanium samples were probed, while Fig.6b. shows the state after probing. EDX analysis of this debris was not able to detect any titanium present indicating the debris was a result of surface contamination transfer to the stylus tip, rather than pickup of Titanium from the sample.

\section{DISCUSSION}

Significant quantities of stylus tip debris were built up on all the probe sensors tested during the course of this work. This is despite the prepared test samples being well cleaned using a combinations of cleaning solutions in an ultrasonic bath. While small quantities of surface contamination were present on the material samples, composition analysis of the contaminated stylus tips indicated the elemental composition of much of the debris to be consistent with the material being probed. This overall observation indicates that stylus tip contamination is a significant problem, with contamination sources arising from both the material being probed as well as other undetermined sources such as surface contamination transfer from the sample to the stylus tip. Even after applying long cleaning cycles which achieved outwardly clean samples as observed to the naked eye, there is still significant potential for stylus tip contamination.

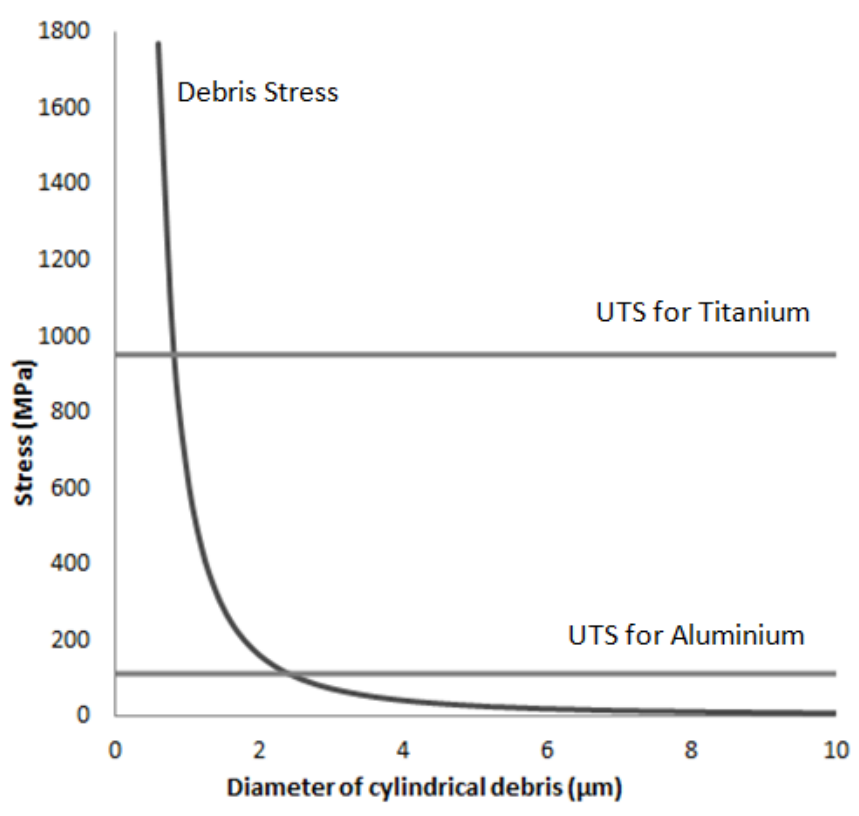

Fig.7. Graph of stress developed in an idealized piece of cylindrical debris adhered to a stylus tip surface subjected to a probing force of $0.5 \mathrm{mN}$.

The size of potential errors caused by stylus tip contamination will depend on a number of factors such as the size and the mechanical properties of debris concerned. For example small soft materials are more likely to be compressed flat under the influence of the applied probing force, whereas larger hard materials will not. In the latter case if the debris lies in a position coincident with the desired contact point of the stylus tip during a given probing operation then a consistent bias will result in all measurements carried out at this point, the size of which relates to the size of the debris less any compression or plastic deformation as a result of probing.

In practice for debris larger than a few micrometers in diameter the resultant compressive stress will have negligible effect on the geometry of the debris. To illustrate the effects of debris size that is likely to cause a problem an idealized cylindrical piece of debris is considered. For a probing force of $0.5 \mathrm{mN}$ the stress generated in the cylinder assuming and evenly distributed load on the top surface of the cylinder is illustrated on the chart shown in Fig.7. On this chart the stress generated in the debris is plotted as a function of the diameter of the cylinder. The chart also indicates the ultimate tensile strength (UTS) for Titanium and Aluminium for illustrative purposes. From the chart it may be seen that for small pieces of debris that are less than $1 \mu \mathrm{m}$ in diameter even for high strength materials the resulting forces will cause significant compression or damage to the debris. However for larger debris greater than around $3 \mu \mathrm{m}$ in diameter the resulting stress is unlikely to have a significant impact even for low strength materials. Observations of the contamination seen in this work suggest debris may range in size from a few micrometers up to tens of micrometers which indicates the significant impact contamination may have on probing accuracy.

The influence of the material being probed on the level of stylus tip contamination was clearly demonstrated by the test results. It was clear that surface finish contributes to the level of stylus tip contamination, as in the case of stylus tip scanning of smooth and rough copper samples there was significant build up of debris following scanning of the rougher material. However, for high strength materials such as Titanium and Stainless Steel material related stylus tip contamination was seen only in trace amounts, as compared to the lower strength materials such as Nylon, Copper, and Aluminium. Even in the case of highly structured Titanium samples, there was no evidence of material transfer from the material to the stylus tip. There was however significant contamination following probing of these rough samples due to transfer of other unknown contamination sources. A possible explanation of this is that the highly structured nature of these surfaces makes them more prone to build-up and trapping of surface contamination that is less easily removed in standard ultrasonic cleaning, and as such represents a greater risk of contamination transfer during probing.

The final conclusion drawn from the experiments is that the probing method may significantly influence the build-up of stylus tip debris. Scanning was seen to results in an increased rate of stylus tip debris build-up, this may be a results of the increased lateral forces due to sliding friction that will be exerted on the work piece, leading to a combination of normal and lateral forces impacted on the probed surface.

With this new knowledge increased awareness of the likelihood of stylus tip contamination has been made 
possible. However, it also suggests there is a significant need for effective cleaning strategies to remove stylus tip contamination. The fragile nature of micro probe sensors makes effective cleaning of these devices a significant challenge. Initial work has been undertaken by the authors to investigate stylus tip cleaning methods and is described elsewhere [11], where a review of cleaning systems that were evaluated by use of SEM surface analysis to examine contamination on stylus tips following each cleaning process is presented. In addition a Clean Logix SP2000 snow-jet cleaning system was shown to remove particulate debris from stylus tips. This process generates a Nitrogen gas jet within which particles of solid Carbon dioxide are mixed and accelerated to the velocity of the Nitrogen gas jet. As the Carbon dioxide particles strike the surface to be cleaned, they impart kinetic energy to any debris present which acts to displace it from the surface. While the results of this cleaning method are promising they do not provide a fully optimised cleaning method and therefore further work is required to develop a solution to this challenging problem.

\section{CONCLUSIONS}

This work has considered the factors which contribute to unwanted micro stylus tip contamination. Due to the cost and fragile nature of micro probing systems, and the difficulty of inspection to characterise contamination, a limited set of data points could be collected effectively. While this has not made it possible to produce a statistical analysis of contamination build-up, or demonstrate detailed trends, it has allowed the establishment of practical bounds for the factors affecting micro stylus tip contamination.

It has demonstrated the significant size of debris that may result from modest levels of probing. The results indicate that this debris occurs both from material transfer as well as contamination transfer from the probed surface to the stylus tip. Probe scanning rather than single point probing was shown to result in greater levels of stylus tip contamination, in terms of both material pickup and contamination transfer. The material properties of the surface were shown to influence the transfer of probed material, with high strength materials resulting in minimal material transfer.

Based on these findings it is suggested that to minimize stylus tip contamination parts should be well cleaned prior to probing. As traditional ultrasonic cleaning may not be adequate additional sample preparation steps may need to be developed. Where possible it is recommended that probing be restricted to single point probing only, especially when probing low strength material.

Future work will be required to develop effective cleaning methods for micro probing systems. In addition stylus contamination assessment systems that do not rely on the use of SEM imaging are required.

\section{ACKNOWLEDGEMENT}

The authors would like to acknowledge the assistance provided with the collection of data by Mark Strickland, Jeremy Straw, and Paul Wentworth of the Precision Manufacturing Centre, The University of Nottingham.

\section{REFERENCES}

[1] Liang, Q., Zhang, D., Wang, Y., Coppola, G., Ge, Y. (2013). PM based multi-component F/T sensorsState of the art and trends. Robotics and ComputerIntegrated Manufacturing, 29 (4), 1-7.

[2] Peiner, E., Balke, M., Doering, L. (2007). Slender tactile sensor for high-aspect-ratio micro metrology. In IEEE Sensors, 28-31 October 2007. IEEE, 760-763.

[3] Ping, Y., Tomohiko, T., Satoru, T., Kiyoshi, T., Osamu, S., Sonko, O., Toshiyuki, T. (2011). Development of high-precision micro-coordinate measuring machine: Multi-probe measurement system for measuring yaw and straightness motion error of XY linear stage. Precision Engineering, 35 (3), 424430.

[4] Weckenmann, A., Estler, T., Peggs, G., McMurtry, D. (2004). Probing systems in dimensional metrology. In CIRP Annals - Manufacturing Technology, 53 (2), 657-684.

[5] Meli, F., Küng, A. (2007). AFM investigation on surface damage caused by mechanical probing with small ruby spheres. Measurement Science and Technology, 18, 496-502.

[6] Nicolet, A., Kung, A., Meli, F. (2012). Study of sapphire probe tip wear when scanning on different materials. Measurement Science and Technology, 23, 094016.

[7] Stone, J., Muralikrishnan, B., Sahay, C. (2011). Geometric effects when measuring small holes with micro contact probes. Research of the National Institute of Standards and Technology, 116, 573-587.

[8] Muralikrishnan, B., Stone, J., Stoup, J. (2006). Fiber deflection probe for small hole metrology. Precision Engineering, 30, 154-164.

[9] Stone, J., Muralikrishnan, B., Stoup, J. (2005). A fiber probe for CMM measurements of small features. In Recent Developments in Traceable Dimensional Measurement III. Proc. SPIE 5879, 254-264.

[10] Wozniak, A., Mayer, R., Balazinski, M. (2009). Stylus tip envelop method: Corrected measured point determination in high definition coordinate metrology. International Journal of Advanced Manufacturing Technology, 42, 505-514.

[11] Kinnell, P., Habeb, R. (2013) An evaluation of cleaning methods for micro-CMM probes. Measurement Science and Technology, 24, 085603.

Received July 25, 2013. Accepted November 29, 2013. 